\title{
Cell-free DNA and circulating TERT promoter mutation for disease monitoring in newly-diagnosed glioblastoma
}

\author{
Maxime Fontanilles ${ }^{1,2,10^{*}}$ (D) Florent Marguet ${ }^{3,4}$, Ludivine Beaussire ${ }^{1}$, Nicolas Magne ${ }^{5}$, Louis-Ferdinand Pépin ${ }^{6}$, \\ Cristina Alexandru², Isabelle Tennevet ${ }^{2}$, Chantal Hanzen ${ }^{7}$, Olivier Langlois ${ }^{8}$, Fabrice Jardin ${ }^{3}$, Annie Laquerrière ${ }^{3,4}$, \\ Nasrin Sarafan-Vasseur ${ }^{1}$, Fréderic Di Fiore ${ }^{1,2,9 \dagger}$ and Florian Clatot $^{1,2+}$
}

\begin{abstract}
The clinical implications of plasmatic cell-free and tumor DNA (cfDNA and ctDNA) are challenging in glioblastoma. This prospective study included 52 consecutive newly diagnosed glioblastoma $(n=49)$ or gliosarcoma $(n=3)$ patients treated with concomitant temozolomide and radiotherapy (RT-TMZ), followed by a TMZ maintenance phase. Plasma samples were collected at baseline, before RT-TMZ (pre-RT-TMZ) and at the end of adjuvant TMZ, or at the time of progression in cases of progressive disease (PD). The cfDNA concentration was measured with a fluorometric method, and ctDNA was detected using targeted droplet digital PCR. The main objectives were to analyze the associations between cfDNA and ctDNA measurements during the course of treatment with PD and survival. There was a significant decrease in median cfDNA concentration from baseline to pre-RT-TMZ-19.4 versus $9.7 \mathrm{ng} / \mathrm{mL}$ $(p<0.0001)$ - in the entire cohort. In patients with PD, a significant increase in cfDNA concentration from pre-RT-TMZ to time of PD was observed, from 9.7 versus $13.1 \mathrm{ng} / \mathrm{mL}(p=0.037)$, respectively, while no difference was observed for nonprogressive patients. Neither the cfDNA concentration at baseline nor its kinetics correlated with survival. ctDNA was detected in 2 patients (3.8\%) and only in gliosarcoma subtypes.

Trial registration ClinicalTrial, NCT02617745. Registered 1 December 2015, https://clinicaltrials.gov/ct2/show/NCT02 617745?term $=$ glioplak\&draw $=2 \&$ rank=1.
\end{abstract}

Keywords: Glioblastoma, Cell-free DNA, Circulating tumor DNA, TERT promoter mutation, Liquid biopsy

\section{Introduction}

Glioblastoma is the most frequent primary adult brain tumor, with 125,000 to 150,000 new cases per year worldwide [1]. First-line treatment for glioblastoma is based on surgical resection whenever possible, followed by radiotherapy (RT) plus concomitant and adjuvant temozolomide (TMZ) [2]. The prognosis remains poor, with a median overall survival time of less than 20 months.

\footnotetext{
*Correspondence: maxime.fontanilles@chb.unicancer.fr

${ }^{\dagger}$ Fréderic Di Fiore and Florian Clatot have contributed equally to this work.

${ }^{10}$ Département d'oncologie médicale, Centre de Lutte Contre le Cancer Henri Becquerel, Rue d'Amiens, 76038 Rouen, France

Full list of author information is available at the end of the article
}

Radiological evaluation using magnetic resonance imaging (MRI), including perfusion and diffusion-weighted sequences, is considered to be the most appropriate method for both tumor burden evaluation and disease monitoring. The diagnosis of recurrence is often challenging because pseudo progression may also occur. A reliable definition of patients with progressive and nonprogressive disease is required both for clinical decisionmaking and for evaluating responses within clinical trials.

In this context, the use of circulating cell-free DNA (cfDNA) is considered very promising to optimize the management of these patients. cfDNA is related to cell turnover, combining both circulating normal DNA original author(s) and the source, provide a link to the Creative Commons licence, and indicate if changes were made. The images or other third party material in this article are included in the article's Creative Commons licence, unless indicated otherwise in a credit line to the material. If material is not included in the article's Creative Commons licence and your intended use is not permitted by statutory regulation or exceeds the permitted use, you will need to obtain permission directly from the copyright holder. To view a copy of this licence, visit http://creativecommons.org/licenses/by/4.0/. The Creative Commons Public Domain Dedication waiver (http://creativeco mmons.org/publicdomain/zero/1.0/) applies to the data made available in this article, unless otherwise stated in a credit line to the data. 
and tumor DNA (ctDNA). The cfDNA concentration is reportedly associated with prognosis in many cancers, while ctDNA, which harbors somatic alterations, was shown to be a surrogate marker for disease monitoring $[3,4]$. As yet, the clinical relevance of these markers is not clearly established in patients with glioblastoma because, in addition to technological considerations, the presence of the blood-brain barrier physically limits the crossing of small genetic fragments [5]. The literature is conflicting regarding the role of cfDNA and ctDNA in glioblastoma. Indeed, although these markers are frequently reported to be informative in cerebrospinal fluid (CSF), their detection rate in plasma has been variable in several studies, ranging from 7.9 to $55 \%$ [6, 7]. Glioblastoma harbors multiple recurrent somatic alterations within epithelial growth factor receptor (EGFR), phosphatase and tensin homolog (PTEN), TP53 or telomerase reverse transcriptase (TERT) genes [8]. TERT encodes a subunit of the telomerase complex. Somatic mutations within the $T E R T$ promoter (TERTp) region are involved in oncogenesis in various type of tumor including glioblastoma [9]. Two recurrent mutations of TERTp that reach $70-80 \%$ frequency in glioblastoma $[10,11]$ are located -124 bp and $-146 \mathrm{bp}$ upstream of the TERT translation start site (chromosome 5p15.33: 1,295,228 $\mathrm{C}>\mathrm{T}$ and 1,295,250 $\mathrm{C}>\mathrm{T}$, respectively $\mathrm{C} 228 \mathrm{~T}$ and $\mathrm{C} 250 \mathrm{~T}$ ). Thus, TERTp mutations may be a potential biomarker for diagnosis [12] and disease monitoring in glioblastoma.

In this context, the aim of this prospective study was to evaluate the cfDNA concentration and the detection rate of ctDNA at diagnosis and during the course of treatment, as well as their correlation with tumor progression, in patients treated for a newly diagnosed glioblastoma.

\section{Materials and methods}

\section{Study population, tumor and plasma collection}

Patients with newly diagnosed, histologically confirmed, supratentorial glioblastoma or gliosarcoma, according to the World Health Organization classification 2016 [13], were prospectively included in our study from July 2016 to November 2017. Patients had to be at least 18 years of age and planned to receive RT (60 Gy in 30 fractions) with concomitant TMZ therapy, followed by adjuvant TMZ with or without previous radical surgery [2]. Patients with concomitant or previous history of cancer within 2 years were excluded. Blood samples were prospectively collected according to the following schedule: at baseline before resection or before biopsy in cases of nonresected tumor; before the initiation of RTTMZ (pre-RT-TMZ); and at the end of adjuvant TMZ treatment, corresponding to the 6th or the 12th cycles according to the physician's choice. In cases of progressive disease (PD), a blood sample was collected at the time of progression, before the end of adjuvant TMZ. All patients were followed according to the RANO criteria [14] using brain MRI every 3 months from the end of the RT-TMZ phase.

All MRIs performed at baseline were reviewed for tumor volume determination. They were analyzed on a T2-Flair and T1 postcontrast sequences after manual segmentation using Advantage Windows $4.6^{\circledR}$ (General Electric Healthcare, Milwaukee, Wisconsin, United States). The analysis of tumor volume was performed in $\mathrm{T} 1$ postcontrast sequences according necrotic areas. The maximal relative cerebral blood volume (rCBV) was also measured by perfusion-weighted imaging, when available, by drawing several regions of interest in high-rCBV areas in the tumor and was compared to the contralateral non-affected brain parenchyma $[15,16]$.

Samples of $6 \mathrm{~mL}$ of whole blood were collected at three different time points into tubes containing ethylenediaminetetraacetic acid (EDTA) based on previous studies $[17,18]$. Within $2 \mathrm{~h}$ after blood collection, the tubes were centrifuged at $3000 \mathrm{rpm}$ for $10 \mathrm{~min}$; the plasma was then extracted and stored at $-80^{\circ} \mathrm{C}$ until use. Tumor samples were obtained during diagnostic procedures (resection or biopsy). After collection, the tumor samples were used for routine histopathology, immunohistochemistry and molecular biology analyses (Additional file 1). Another tumor sample was flash-frozen at $-80{ }^{\circ} \mathrm{C}$ and stored until use. Ten healthy subjects were included as plasma sample controls. The study was an ancillary of the ongoing prospective GLIOPLAK trial, registered in ClinicalTrials.gov (NCT02617745, https://clinicaltrials.gov/ct2/ show/NCT02617745). Informed written consent to participate in the study was obtained from all patients, and the French National Committee for the Protection of Persons approved the study (RCB ID 2015-A00377-42).

\section{Cell-free DNA extraction and quantification}

cfDNA was extracted from 1 to $5 \mathrm{~mL}$ of plasma using the QIAamp Circulating Nucleic Acid kit ${ }^{\circledR}$ (Qiagen, Hilden, Germany) according to the manufacturer's instructions. The sample was eluted in a final volume of $30 \mu \mathrm{L}$ and stored at $-20{ }^{\circ} \mathrm{C}$. Double-stranded DNA quantification was performed by a fluorometric method using a Quantit ${ }^{\mathrm{TM}}$ PicoGreen ${ }^{\circledR}$ dsDNA Assay kit (Invitrogen, Carlsbad, CA, USA) and a Twinkle LB970 microplate fluorimeter (Berthold, Bad Wildbad, Germany). For each sample, cfDNA quantification was performed in duplicate from $2 \mu \mathrm{L}$ of eluate, and normalization was performed using a standard calibration curve of known concentrations of standard dsDNA (from 0 to $10 \mathrm{ng}$ ).

The fragment distribution of the cfDNA was analyzed using the Agilent 4200 TapeStation ${ }^{\circledR}$ System (Santa Clara, 
CA USA) with D5000 High-Sensitivity ScreenTape. This system uses electrophoresis to separate DNA fragments from 100 to 5000 base pairs (bp). The mean DNA length is then automatically estimated for each peak.

\section{Circulating tumor DNA exploration by droplet digital PCR} ctDNA detection was analyzed according to telomerase reverse transcriptase promoter (TERTp) mutation detection by droplet digital PCR (ddPCR) (Additional file 1). The limit of detection (LOD) was determined from the plasma of healthy subjects according to a previously reported method [12, 13]. Healthy subjects were volunteers free from acute or chronic pathology; plasma samples were collected and stored under the same preanalytical conditions as for the patients. Short-sized TERTp sequencing was performed using a ddPCRbased method with an amplicon of 88 bp (reference dHsaEXD20945488 for TERTp C228T and reference dHsaEXD85215261 for TERTp C250T). The experimental procedure was identical to that used for the $113 \mathrm{bp}$ assays. Positive-case control was a patient suffering from TERTp-mutated hepatocellular carcinoma.

Matched tumor DNA was extracted from formalinfixed paraffin-embedded tissue and sequenced using ddPCR $113 \mathrm{bp}$ assay following the previously-described procedure.

\section{Statistical analyses}

The objective was to evaluate variations of cfDNA and ctDNA and their associations with baseline characteristics and outcome. The analysis was performed in the whole population and according to the presence (or not) of a resected tumor. The outcome was analyzed according to the rate of PD at 6 and 12 months, as well as progression-free survival (PFS) and overall survival (OS). OS was defined as the time from diagnosis to the date of death or last follow-up. PFS was defined as the time from diagnosis to the date of first disease progression or date of death. Prognostic factors such as the Karnofsky performance status index, type of surgical procedure (biopsy versus resection) and O6-methylguanine-DNA methyltransferase promoter (MGMTp) methylation status were explored in univariate and multivariate analyses. OS and PFS were calculated using Kaplan-Meier method and compared with the log-rank test. Multivariate analyses were performed using variables in univariate analyses with a $P$ value less than or equal to $10 \%$. Follow-up period for survival analyzes was set at 12 months; in case of nonprogressive disease monitoring continued up to 2 years (median 4 months). The data were compared using Student's test for normally distributed continuous variables or the Mann-Whitney-Wilcoxon test for nonparametric variables; the Fisher exact test or Pearson's Chi squared test was used for categorical variables, as appropriate. A difference was considered statistically significant if the degree of significance ( $p$ value) was less than or equal to 0.05 (alpha risk $=5 \%$ ). Statistical analyses were performed using the $\mathrm{R}$ software ( $\mathrm{R}$ version 3.5.1, 2018, Vienna, Austria) [19].

\section{Results}

\section{Patients' characteristics}

A total of 52 patients were included. As shown in Table 1, $62 \%(32 / 52)$ underwent surgical resection of the primary tumor, and all patients underwent an RT-TMZ sequence. Based on a median follow-up of 12 months (2-25 months), 41 patients (78\%) had PD and 28 died (54\%). Among patients with PD, 30 patients (58\%) had progression during the TMZ maintenance phase. The

Table 1 Clinical and tumor characteristics

\begin{tabular}{ll}
\hline Characteristics & Number of patients (\%) \\
\hline Age (years), mean \pm SD & $58.3 \pm 9.5$ \\
Sex & \\
Female & $22(42.3 \%)$ \\
Male & $30(57.7 \%)$ \\
Surgery & \\
Biopsy & $20(38.4 \%)$ \\
Resection & $32(61.6 \%)$ \\
Karnofsky performance score & \\
$\geq 80 \%$ & $45(86.5 \%)$ \\
$\geq 70$ to <80\% & $6(11.5 \%)$ \\
$<70 \%$ & $1(2 \%)$ \\
Tumor Characteristics & \\
Volume at baseline, mean \pm SD & \\
T1-weighted with Gadolinium W/necrosis & $31.1 \mathrm{~cm}^{3} \pm 28.6$ \\
T1-weighted with Gadolinium W/O necrosis & $18 \mathrm{~cm}^{3} \pm 16.5$ \\
Necrosis part, mean (\%) & $33 \%[\mathrm{~min} .0-$ max. $87 \%]$ \\
Flair & $114.9 \mathrm{~cm}^{3} \pm 63.2$ \\
Histology & \\
Glioblastoma-IDH wild type & $46(88.4 \%)$ \\
Glioblastoma IDH-mutated & $3(5.8 \%)$ \\
Gliosarcoma & $3(5.8 \%)$ \\
MGMTp methylation & \\
Non-methylated & $26(50 \%)$ \\
Low methylation profile & $2(4 \%)$ \\
Methylated & $12(23 \%)$ \\
Unknown & $12(23 \%)$ \\
TERTp mutation & \\
C228T & $44(84.6 \%)$ \\
Wild-type (wt) & $1(1.9 \%)$ \\
Unknown & \\
\hline
\end{tabular}


median PFS was 8.5 months [95\% confidence interval 6-13], and the median OS was 17 months [95\% CI 16-21]. Blood samples were available for $90 \%$ of the patients (47/52) at baseline, for $100 \%$ of the patients (52/52) before RT-TMZ initiation, and for $90 \%$ of the patients $(47 / 52)$ for the third sample, 5 patients died before the third blood collection. The third sample was collected from 47 patients either at the 6 th $(n=10)$ or at 12 th TMZ cycle $(n=12)$ or at the time of PD $(n=25)$.

\section{Baseline cfDNA}

The median cfDNA concentration was $19.4 \mathrm{ng} / \mathrm{mL}$ (interquartile range (IQR) 13.6-27.6) compared to $5.6 \mathrm{ng} /$ $\mathrm{mL}$ (IQR 5.1-6.6) in the group of 10 healthy volunteers, $p<0.0001$. The cfDNA concentration has a significant although weakly positive association with the T1-weighted gadolinium-enhanced volume without necrosis $\left(\mathrm{R}^{2} 0.12, p=0.018\right)$ but not with the FLAIR volume or the T1-weighted gadolinium-enhanced volume with necrosis (Fig. 1c, a, b respectively). The $\mathrm{rCBV}$ was measured for 27 patients (mean $=9.03$ [range 4-24]) and correlated with the cfDNA concentration (R-squared $0.32, p=0.03$ ) (Fig. 1d). The cfDNA tended to be higher in patients treated with corticosteroid $(n=30)$ compared to patients who were not $(\mathrm{n}=17)(21 \mathrm{ng} / \mathrm{mL}$ versus $14.4 \mathrm{ng} / \mathrm{mL}(p=0.1)$, respectively). The level of cfDNA was not correlated with CRP $\left(\mathrm{R}^{2} 0.001, p=0.71\right)$ or albuminemia $\left(\mathrm{R}^{2} 0.005, p=0.5\right)$.

\section{Correlations between cfDNA variations during treatment and clinical outcome}

A significant decrease in median cfDNA concentration was observed from baseline to pre-RT-TMZ, $19.4 \mathrm{ng} / \mathrm{mL}$ versus $9.7 \mathrm{ng} / \mathrm{mL}(p<0.0001)$ respectively, corresponding to a mean decrease of $-53 \%$ (range -94 to $-1 \%$ ). The cfDNA decrease was observed both in the biopsy group and in the resection group $(16.9 \mathrm{ng} / \mathrm{mL}$ vs. $10.5 \mathrm{ng} / \mathrm{mL}$ $(p=0.022)$ and $20.4 \mathrm{ng} / \mathrm{mL}$ vs $9.4 \mathrm{ng} / \mathrm{mL},(p<0.001)$, respectively). Decrease in corticosteroid exposure was observed between baseline and pre-RT-TMZ; and could explain the cfDNA decrease in the entire cohort: mean equivalent prednisolone dose of $63 \mathrm{mg}$ at baseline versus $32 \mathrm{mg}$ at pre-RT-TMZ $(p=0.01)$. Thus, a non-significant decrease in corticosteroid dose in the biopsy group (54 $\mathrm{mg}$ at baseline vs. $42 \mathrm{mg}$ at pre-RT-TMZ, $p=0.43$ ) could partly explain the decrease of cfDNA in the biopsy group.

Among the 22 patients without PD during the adjuvant TMZ phase, no difference in the cfDNA concentrations was observed between pre-TMZ and the end of the adjuvant TMZ phase (9.7 vs. $9.5 \mathrm{ng} / \mathrm{mL}(p=0.6)$, respectively). In contrast, among the 30 patients who experienced PD during the TMZ phase, we observed a significant increase in the median cfDNA level from pre-TMZ to the time of progression (9.7 vs. $13.1 \mathrm{ng} / \mathrm{mL}$ $(p=0.037)$, respectively) (Additional file 2 : Table S1). The increase in cfDNA was mainly observed among patients with biopsy only, $10.5 \mathrm{ng} / \mathrm{mL}$ versus $21.1 \mathrm{ng} /$ $\mathrm{mL}(p=0.019)$ (Fig. 2), and was independent of corticosteroid dose adjustment, with a mean equivalent prednisolone dose of $42 \mathrm{mg}$ at pre-RT-TMZ versus $51 \mathrm{mg}$ at progression $(p=0.73)$.

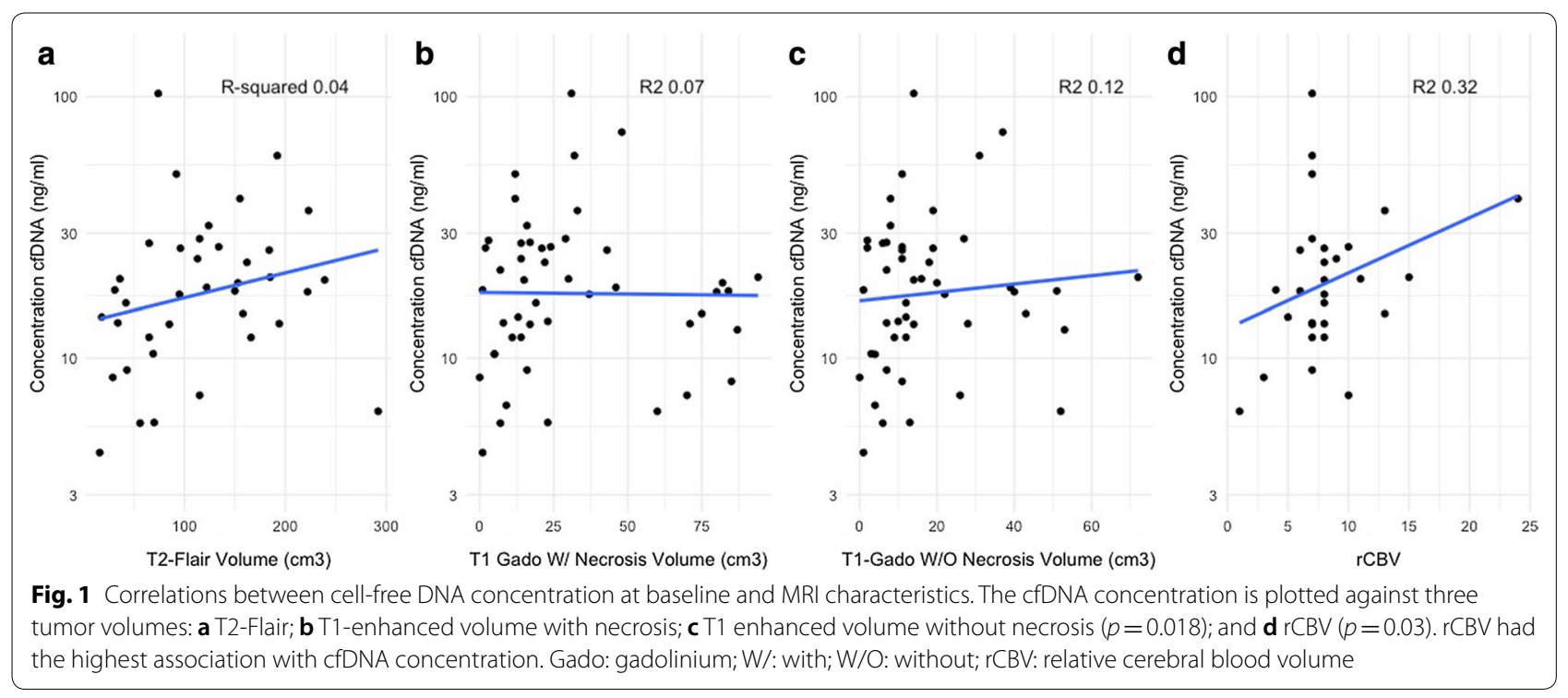



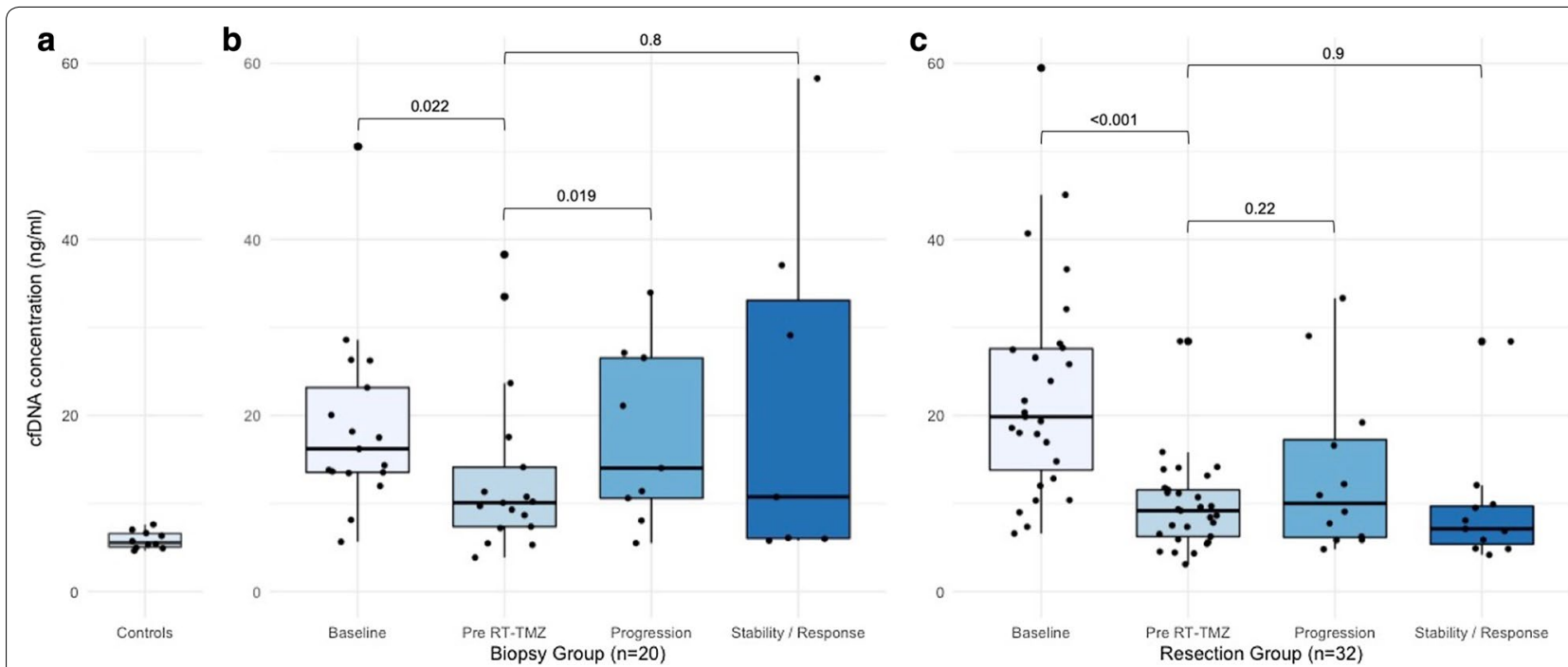

Fig. 2 Variations of circulating cell-free DNA concentration during treatment. a Median cfDNA concentration ( $\mathrm{ng} / \mathrm{mL}$ of plasma) at baseline is higher than in the control group (healthy subjects) and decreases between baseline and pre-RT-TMZ in both the biopsy group (b) and the tumor resection group (c). RT: radiotherapy; TMZ: temozolomide

In the entire cohort, the baseline cfDNA concentration and its variation before the RT-TMZ phase were not associated with OS or PFS (Fig. 3, Additional file 3: Table S2). In the subgroup of patients with resection, the baseline cfDNA concentration and its kinetics before the RT-TMZ phase did not influence overall survival or progression-free survival (data not shown).

The cfDNA size estimation by the TapeStation ${ }^{\circledR}$ method was performed for $51 / 52$ patients for at least one of the three time point samples. Overall, the median DNA size was 86 bp [IQR 67.5-596], with $63 \%$ of the DNA fragments less than $113 \mathrm{bp}$. No difference in DNA size according to the timing of samples was observed (Additional file 2: Table S1). There was no association between DNA size and cfDNA concentration.

\section{ctDNA detection using TERTp mutation}

Plasma samples from 46 patients $(46 / 52,88.4 \%)$ were available for ctDNA TERTp C228T and C250T $113 \mathrm{bp}$ ddPCR assays. Among them, 2 (2/46, 4.3\%) had a detectable circulating TERTp mutation in at least one sample. The first patient had a circulating TERTp C228T mutation detected at baseline and at pre-RT-TMZ (Fig. 4). The second patient had TERTp C228T detected only at time of PD, when distant metastases were diagnosed (Fig. 5). Interestingly, TERTp detection in plasma seemed to be restricted to the gliosarcoma subtype: $2 / 3(67 \%)$ versus $0 / 49$ for glioblastoma. TERTp mutation analysis using the ddPCR 88 bp assay did not increase the detection rate. Interestingly, 2 plasma samples $(3.8 \%)$ harbored at least 2 droplets using a duplicate 88 bp assay and were considered as negative according to the positive threshold (Additional file 4: Table S3, Additional file 5: Figure S1).

\section{Discussion}

Our results, based on serial blood samples collected prospectively, suggest that the evaluation of cfDNA concentration, in contrast to TERTp mutation from plasmatic ctDNA, may give clinically relevant information in patients treated with RT-TMZ for glioblastoma. At baseline, we observed that the cfDNA level was associated with tumor neoangiogenesis based on MRI parameters (R-squared 0.32 regarding association between $\mathrm{rCBV}$ and cfDNA concentration). Our results also highlighted that cfDNA underwent significant variation during the treatment course, with a significant decrease after initial surgery or biopsy procedures and a significant increase in cases of PD during the TMZ maintenance phase. As yet, the role of cfDNA in patients treated for glioblastoma has not been clearly established. In a subgroup of 12 patients, Bagley et al. recently reported that cfDNA increased in cases of PD and remained stable in nonprogressive patients, similar to our findings [7]. Moreover, the interest of cfDNA as a reflection of tumor burden was previously reported in a series showing that its level may correlate with radiological parameters. Taken together, these findings support the hypothesis that cfDNA is released from the vascularized part of the tumor [20] and that this marker might be a useful tool for disease monitoring in the TMZ maintenance phase. 

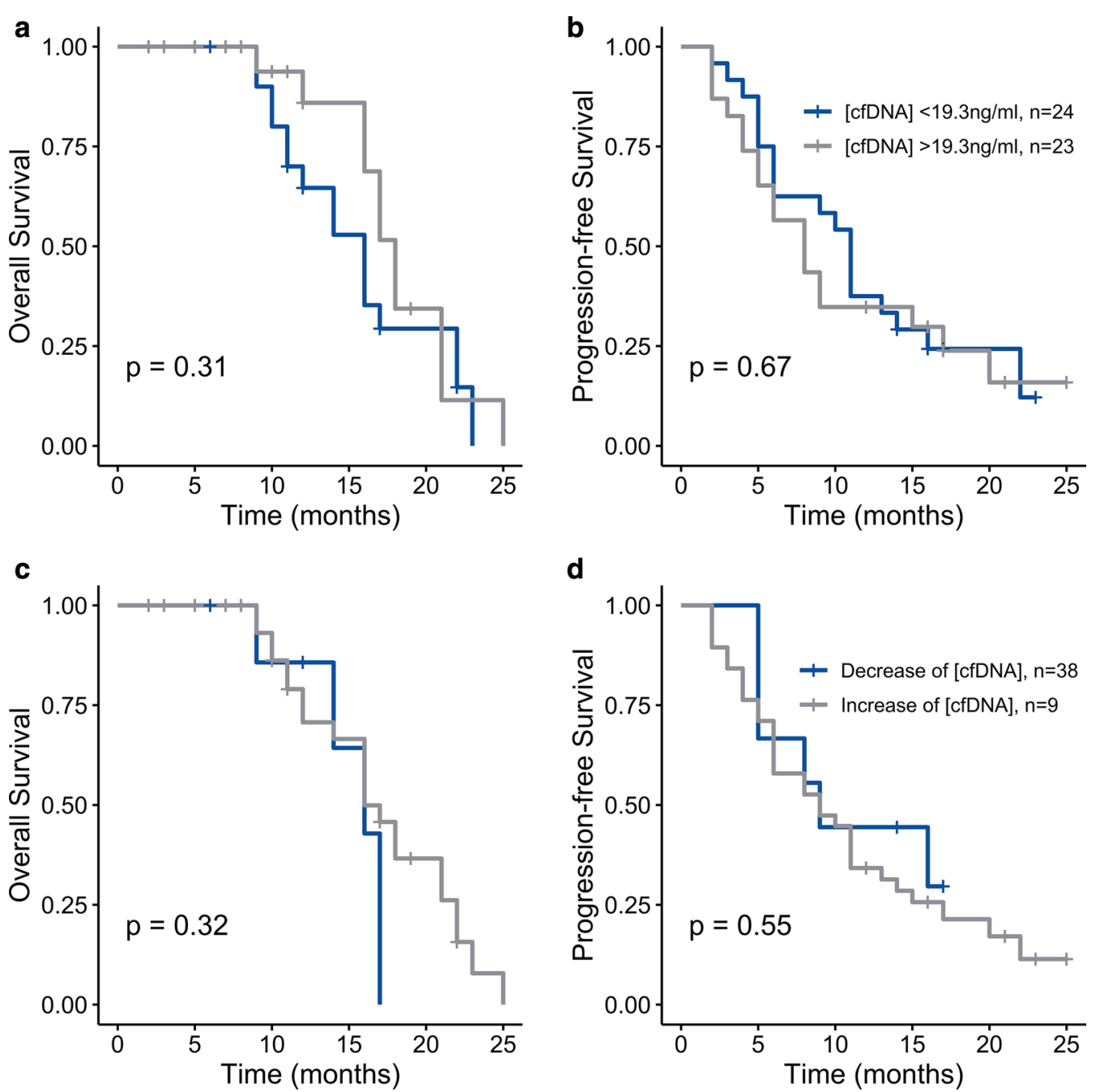

Fig. 3 Survival curves. a Overall survival and progression-free survival $\mathbf{b}$ according to baseline cfDNA concentration below or above the median. OS (c) and PFS (d) according to the increase or decrease of cfDNA between baseline and pre-RT-TMZ

The evaluation of plasmatic ctDNA has been rarely investigated in prospective studies of patients with glioblastoma treated with RT-TMZ. In our work, only 2 cases with detectable circulating TERTp mutation $(\mathrm{n}=2 / 52$, $3.8 \%$ ) were observed using ddPCR, whereas $85 \%$ of the tumors were mutated, which was in accordance with previous studies $[11,21]$. The TERTp mutation detection rate was similar to that of Juratli et al., showing a rate at baseline of $7.9 \%$ by nested PCR in 38 patients [6]. ddPCR was used for TERTp mutation detection, because this sequencing method is an ultrasensitive, fast and costeffective tool, enabling its routine use in clinical practice. The lack of detected TERTp mutations in plasma could be related to the short size of the ctDNA fragments ( $<70 \mathrm{bp})$, which are not able to be detected by current sequencing methods [22]. ddPCR is a sensitive method for detecting DNA fragments with amplicon sizes of $113 \mathrm{bp}$ and $88 \mathrm{bp}$ for TERTp C228T and TERTp C250T. Unfortunately, the use of the 88 bp amplicon size did not increase the sensitivity of ddPCR in our study. These results are to be balanced by the fact that some samples presented very few mutated droplets (samples considered as negative regarding our LOD) and that the quantity of DNA used for the 88 bp assay was less than that used for the $113 \mathrm{bp}$ assay. Another point of discussion to increase detection rate would be to increase the supply of ctDNA. In our study, the patients had an average of $6 \mathrm{~mL}$ of whole blood per collection time, which appeared ethically acceptable. The question of increasing the volume of blood collected to increase the detection of ctDNA could be asked in a dedicated methodological study. Although the detection of circulating TERTp mutation is a rare, our 

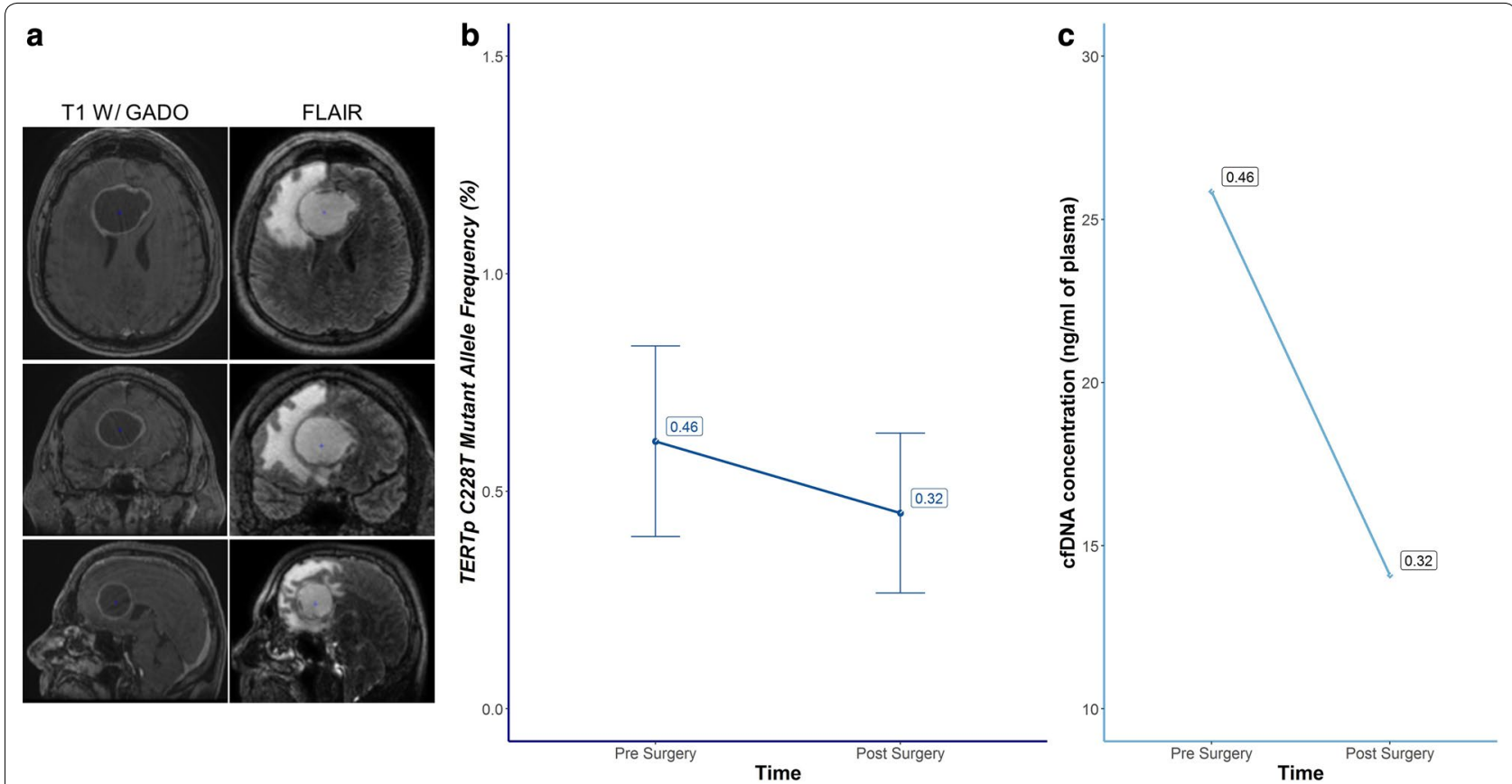

Fig. 4 Patient (\#059) with TERTp C228T gliosarcoma and TERTp detection in plasma at diagnosis. Patient \#059 was a 64-year-old man suffering from frontal C228T TERTp, IDH wild-type gliosarcoma spreading into the corpus callosum. a The lesion was predominantly necrotic: $43 \mathrm{~cm}^{3}$ of T1-weighted gadolinium volume with necrosis versus $11 \mathrm{~cm}^{3}$ without necrosis; the percentage of necrosis was $74 \%$. In contrast, the rest of the cohort had an average rate of necrosis of 33\%. The T2-FLAIR volume was also consequential, with a total volume of $184 \mathrm{~cm}^{3}$. b Circulating C228T TERTp mutation was detected both pre- and postoperatively. Two assays were performed (assay 1 and 2). The presented results are the mean of the two assays, with error bar ( \pm standard deviation). $\mathbf{c}$ The kinetics of cfDNA concentration correlated with ctDNA MAF and decreased after tumor resection

results also underlined that its detection may be of particular interest in two situations: extraneural metastatic dissemination, as previously reported in a case of metastatic ependymoma [12], and the gliosarcoma subtype. Extraneural metastatic dissemination of gliomas is a rare, end-stage event, occurring in less than $2 \%$ of all cases [23] and might be linked to blood-brain barrier rupture with hematogenous dissemination favored by first-line surgery [24]. Unlike glioblastoma, gliosarcoma is characterized by its ability to invade the skull and disseminate systemically [13]. It has been postulated that in cases of gliosarcoma, the blood-brain barrier has increased permeability, with a consequently greater release of ctDNA into the general blood circulation [25]. In contrast to the ddPCR method, the use of targeted next-generation sequencing (NGS) would likely increase the ctDNA detection rate by identifying other recurrent altered genes [7], but this did not correspond to our primary objective which was to identify a single biomarker of disease evolution. The clinical utility, as well as the sensitivity, of ctDNA detection in plasma by ddPCR and the NGS method require further characterization in larger cohorts, especially compared to CSF $[26,27]$. The choice of a non-selected population was made in order to explore the interest of the liquid biopsy concept in the daily-practice of patients with grade IV glioma (resection and biopsy, glioblastoma IDHwt, glioblastoma IDHmut, gliosarcoma). Regarding our results, a prospective study dedicated to the gliosarcoma or bulky IDHwt glioblastoma subgroup would be of major interest.

Finally, the survival analyzes failed to show an impact of cfDNA, in contrast to the survival data reported by Bagley et al. [7]. The survival results should be interpreted with caution due to the lack of power. Prospective and dedicated data are warranted to identify any prognostic significance of the cfDNA baseline concentration. An early cfDNA decrease after a diagnostic procedure should be investigated in a larger homogeneous cohort by separately analyzing resected tumor patients and bulky tumor patients. In addition, it would be interesting to explore the impact on survival of cfDNA kinetics before and right after RT-TMZ phase, before TMZ maintenance phase. The specific impact of corticosteroid therapy on the tumor release of cfDNA remains unclear despite a trend of association observed in our cohort at baseline $(21 \mathrm{ng} / \mathrm{mL}$ vs. $14.4 \mathrm{ng} / \mathrm{mL}(p=0.1))$. There is a high likelihood that the prednisolone equivalent dose and the tumor volume are confounding factors for cfDNA. During TMZ 


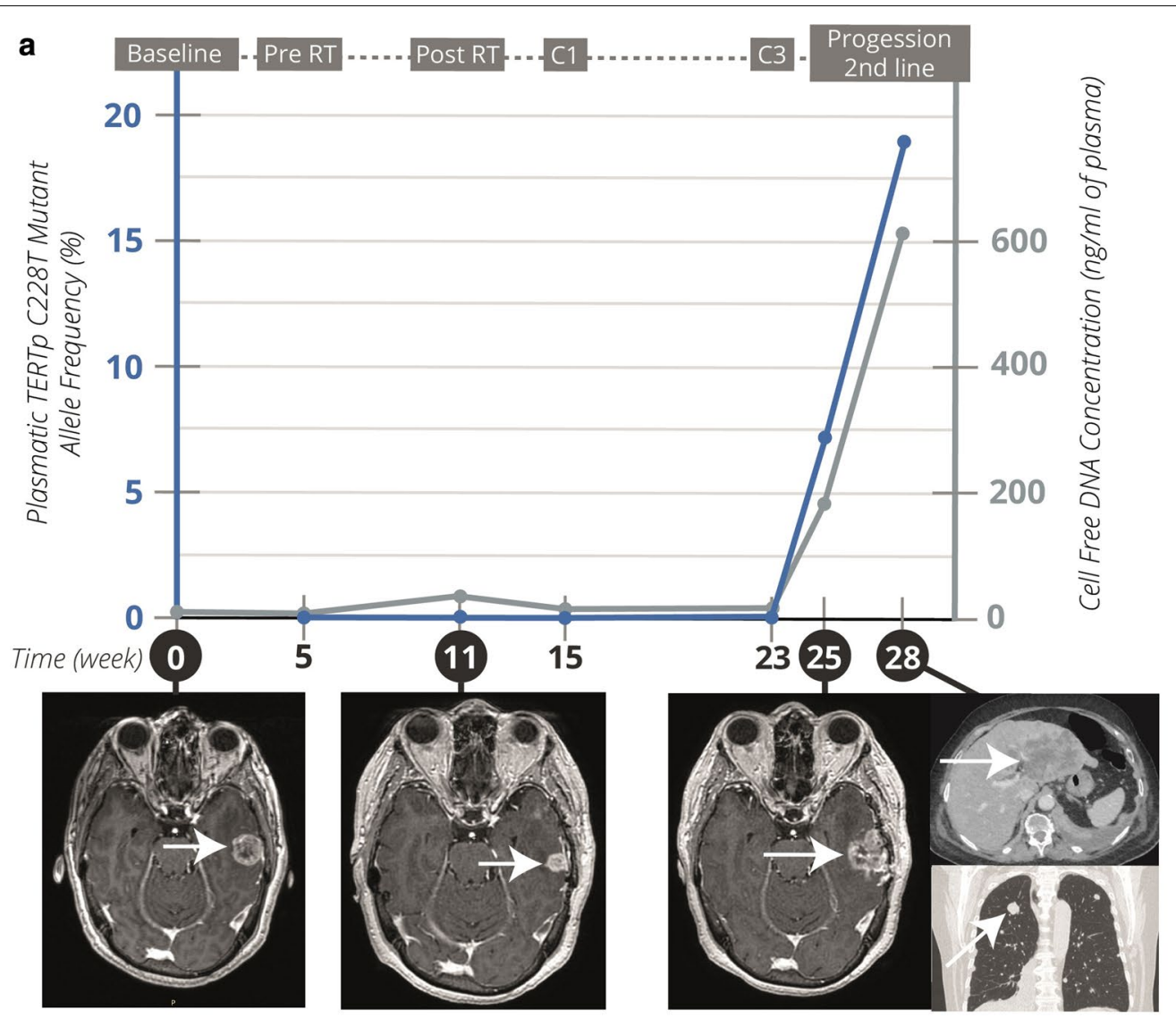

b
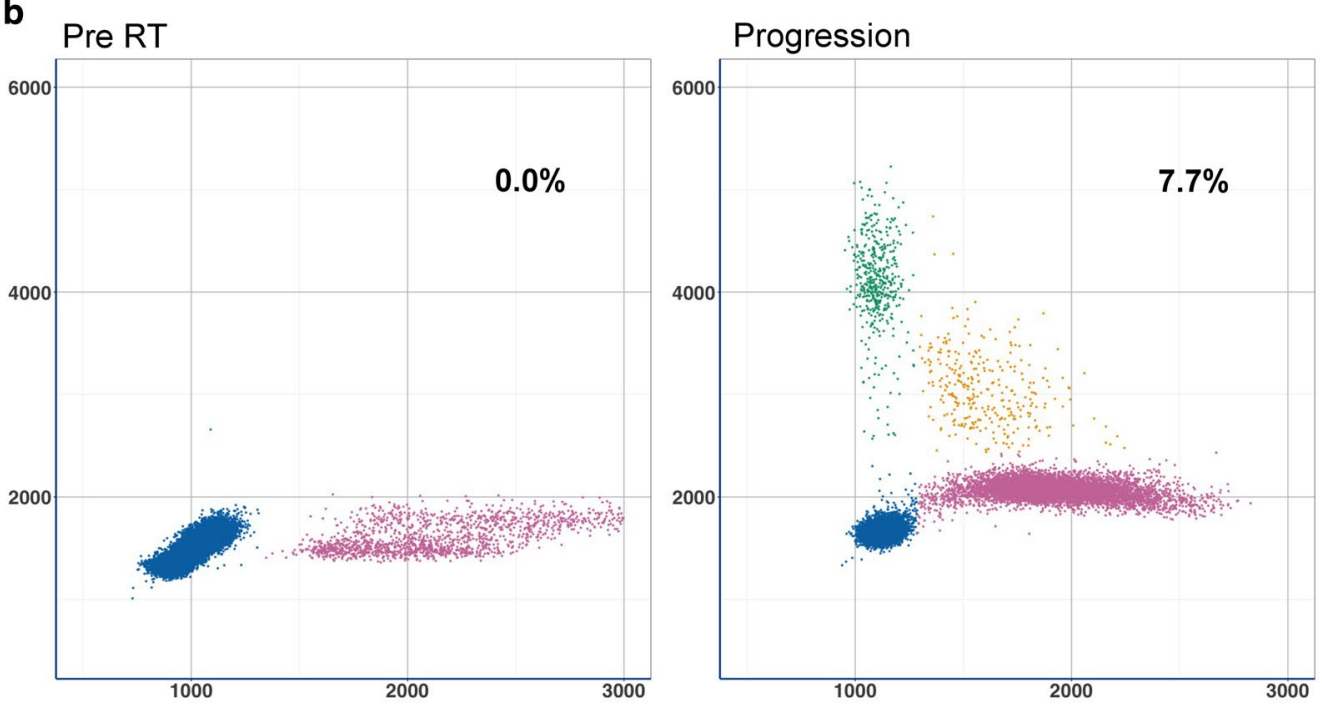

Fig. 5 Plasma longitudinal follow-up of the TERTp C228T mutation in a patient (\#082) with TERTp-mutated gliosarcoma. a Patient \#082 was a 69-year-old woman suffering from left temporal unmethylated MGMTp, IDH wild-type (wt) gliosarcoma with C228T TERTp mutation (MAF 28.6\% in initial resected tumor using ddPCR). Partial tumor resection was initially performed. The plot shows the evolution of MAF and plasma cfDNA concentration as a function of time. C228T TERTp mutation was not detected in plasma until cerebral relapse, with sphenoid bone invasion. The longitudinal plasma samples taken during the first-line treatment schedule revealed a correlation between circulating C228T TERTp MAF, cfDNA concentration, and the evolution towards metastatic status. b Two representations of the search for TERTp C228T mutation in plasma at baseline (left) and at progression (right). Channel 1 fluorescence (vertical axis, FAM ${ }^{\mathrm{TM}}$ ) is plotted against channel 2 (horizontal axis, HEX ${ }^{\mathrm{TM}}$ ). Droplets are grouped as clusters: FAM $^{\mathrm{TM}} / \mathrm{HEX}^{\mathrm{TM}}$ negative (double-negative droplets, blue points), FAM ${ }^{\mathrm{TM}}$ positive/HEX $X^{\mathrm{TM}}$ negative (green points), FAM ${ }^{\mathrm{TM}}$ negative/ HEX ${ }^{\mathrm{TM}}$ positive (pink points), and FAM ${ }^{\mathrm{TM}} / \mathrm{HEX}^{\mathrm{TM}}$ positive (double-positive droplets, orange points) 
treatment, the cfDNA concentration continues to vary according to the tumor evolution-independent of the corticosteroid dose adjustment. Conversely, very high doses of corticosteroid delivered as a bolus could contribute to cfDNA release into the bloodstream and may explain the early cfDNA decrease between biopsy and the pre-RT-TMZ time point in this specific subgroup for whom no oncologic treatment was administered. As previously reported in other cancers, a transient spike of cfDNA may be observed right after tumor procedure [28]. In our cohort we did not investigate this early cfDNA variation due to the lack of blood sample few days after diagnostic procedure. This early variation should be interesting to analyze regarding glioblastoma and biopsy procedure in further studies. No association between cfDNA and CRP, as inflammatory state marker, was observed. However, it would be interesting in further studies to analyze the relationship between cfDNA, corticosteroid, plasmatic inflammatory markers and white blood cell counts.

\section{Conclusion}

Our results showed that, in contrast to ctDNA using TERTp mutation detection, the cfDNA concentration varies significantly over the course of treatment and may be a biomarker of PD during the TMZ phase.

\section{Supplementary information}

Supplementary information accompanies this paper at https://doi. org/10.1186/s40478-020-01057-7.

\section{Additional files}

\section{Additional file 1. Supplementary methods.}

Additional file 2: Table S1. Comparison of cfDNA characteristics at different sampling times.

Additional file 3: Table S2. Results of the Cox model for overall survival and progression-free survival.

Additional file 4: Table S3. Raw data from TERTp C228T and C250T sequencing with $113 \mathrm{bp}$ and $88 \mathrm{bp}$ assays per patient in the entire cohort.

Additional file 5: Figure S1. Analysis of the cfDNA fragment sizes and impact on ctDNA detection. a Control case: A patient suffering from TERTp-mutated hepatocellular carcinoma. Highly sensitive (HS) TapeStation ${ }^{\circledR}$ electrophoresis identified a DNA peak at 132 bp (left). Total cfDNA sequencing confirmed the presence of ctDNA by detecting the circulating TERTp mutation, with an allelic mutation frequency of 19.6\% (right). b Patient with TERTp glioblastoma. HS TapeStation ${ }^{\circledR}$ electrophoresis identified a DNA peak at 80 bp. 113 bp TERTp ddPCR assay did not detect any positive droplet; c whereas 88 bp TERTp ddPCR assay identified positive droplets in duplicate experiments. This case remained negative for circulating TERTP detection regarding the positive threshold due to the very low MAF

\section{Abbreviations}

bp: base pair; cfDNA: cell-free DNA; ctDNA: circulating cell-free tumor DNA; Cl: confidence interval; CSF: cerebrospinal fluid; ddPCR: digital droplet polymerase chain reaction; EDTA: ethylenediaminetetraacetic acid; IDH: isocitrate dehydrogenase; IQR: interquartile range; LOD: limit of detection; MAF: mutant allele frequency; MGMT: O-6-methylguanine-DNA methyltransferase; MRI: magnetic resonance imaging; NGS: next generation sequencing; PFS: progression free survival; PD: progressive disease; OR: odds ratio; OS: overall survival; RANO: response assessment in neuro-oncology; rCBV: relative cerebral blood volume; RT: radiotherapy; TERT: telomerase reverse transcriptase; TMZ: temozolomide.

\section{Acknowledgements}

The authors thank Mrs. Doriane RICHARD for drafting the protocol, for the conduct of the study and for carrying out regulatory procedures; Mrs Sabine LORIVEL for the constitution of biological collection and Mr Anthony MARTINEZ for figure design.

\section{Authors' contributions}

MF was the principal investigator, designed and conceptualized the study, performed data collection and analysis and drafted the manuscript. FM and AL performed diagnostic analyzes revised the manuscript and contributed to the final version. LB performed laboratory analyzes. NS-V performed laboratory analyzes and contributed to the manuscript and data analyzes. NM performed imagery analyzes. L-FP supervised the trial and data/plasmatic collection. CA, IT, CH and OL enrolled patients. FJ supervised study and manuscript redaction. FDF and FC designed, conceptualized the study, supervised data analyzes and drafted final manuscript. All the authors revised and approved the final version of the manuscript.

\section{Funding}

This project was supported by the Institute of Research and Innovation in Biomedicine of Normandy (IRIB, Rouen, Normandie, France) and by Cancer Center Henri Becquerel.

\section{Availability of data and materials}

Deidentified data are available in supplementary material.

\section{Ethics approval and consent to participate}

An informed written consent to participate to the study was obtained from all patients and the French National Committee for the Protection of Persons approved the study (RCB ID 2015-A00377-42).

\section{Consent for publication \\ Not applicable.}

\section{Competing interest}

All the authors report no disclosure.

\section{Author details}

${ }^{1}$ Inserm U1245, Normandie Univ, UNIROUEN, IRON group, Normandy Centre for Genomic and Personalized Medicine, Rouen, France. ${ }^{2}$ Department of Medical Oncology, Cancer Centre Henri Becquerel, Rue d'Amiens, 76000 Rouen, France. ${ }^{3}$ Normandie Univ, UNIROUEN, Inserm U1245, Normandy Centre for Genomic and Personalized Medicine, Rouen, France. ${ }^{4}$ Department of Pathology, Rouen University Hospital, 76031 Rouen, France. ${ }^{5}$ Department of Radiology, Rouen University Hospital, 76031 Rouen, France. ${ }^{6}$ Department of Statistics and Clinical Research Unit, Henri Becquerel Cancer Center, Rouen, France. ${ }^{7}$ Department of Radiation Oncology and Medical Physics, Cancer Centre Henri Becquerel, Rue d'Amiens, 76000 Rouen, France. ${ }^{8}$ Department of Neurosurgery, Rouen University Hospital, 76031 Rouen, France. ${ }^{9}$ Department of Gastroenterology, Rouen University Hospital, 76031 Rouen, France. ${ }^{10}$ Département d'oncologie médicale, Centre de Lutte Contre le Cancer Henri Becquerel, Rue d'Amiens, 76038 Rouen, France.

Received: 26 August 2020 Accepted: 10 October 2020

Published online: 04 November 2020 


\section{References}

1. Ostrom QT, Gittleman H, Liao P, Vecchione-Koval T, Wolinsky Y, Kruchko C et al (2017) CBTRUS statistical report: primary brain and other central nervous system tumors diagnosed in the United States in 2010-2014. Neuro-oncology 19:v1-v88

2. Stupp R, Mason WP, van den Bent MJ, Weller M, Fisher B, Taphoorn MJB et al (2005) Radiotherapy plus concomitant and adjuvant temozolomide for glioblastoma. N Engl J Med 352:987-996

3. Hamfjord J, Guren TK, Dajani O, Johansen JS, Glimelius B, Sorbye H et al (2019) Total circulating cell-free DNA as a prognostic biomarker in metastatic colorectal cancer prior to first-line oxaliplatin-based chemotherapy. Ann Oncol 30:1088-1095

4. Panagopoulou M, Karaglani M, Balgkouranidou I, Biziota E, Koukaki T, Karamitrousis E et al (2019) Circulating cell-free DNA in breast cancer: size profiling, levels, and methylation patterns lead to prognostic and predictive classifiers. Oncogene 38:3387-3401

5. Mair R, Mouliere F, Smith CG, Chandrananda D, Gale D, Marass F et al (2019) Measurement of plasma cell-free mitochondrial tumor DNA improves detection of glioblastoma in patient-derived orthotopic xenograft models. Cancer Res 79:220-230

6. Juratli TA, Stasik S, Zolal A, Schuster C, Richter S, Daubner D et al (2018) TERT promoter mutation detection in cell-free tumor-derived DNA in patients with IDH wild-type glioblastomas: a pilot prospective study. Clin Cancer Res 24:5282-5291

7. Bagley SJ, Nabavizadeh SA, Mays JJ, Till JE, Ware JB, Levy S et al (2020) Clinical utility of plasma cell-free DNA in adult patients with newly diagnosed glioblastoma: a pilot prospective study. Clin Cancer Res 26:397-407

8. Brennan CW, Verhaak RGW, McKenna A, Campos B, Noushmehr H, Salama SR et al (2013) The somatic genomic landscape of glioblastoma. Cell 155:462-477

9. Vinagre J, Almeida A, Pópulo H, Batista R, Lyra J, Pinto V et al (2013) Frequency of TERT promoter mutations in human cancers. Nat Commun 4:2185

10. Nonoguchi N, Ohta T, Oh J-E, Kim Y-H, Kleihues P, Ohgaki H (2013) TERT promoter mutations in primary and secondary glioblastomas. Acta Neuropathol 126:931-937

11. Simon M, Hosen I, Gousias K, Rachakonda S, Heidenreich B, Gessi M et al (2015) TERT promoter mutations: a novel independent prognostic factor in primary glioblastomas. Neuro-oncology 17:45-52

12. Deniel A, Marguet F, Beaussire L, Tobenas-Dujardin A-C, Peillon C, Gambirasio M-A et al (2019) TERTp mutation detection in plasma by dropletdigital polymerase chain reaction in spinal myxopapillary ependymoma with lung metastases. World Neurosurg 130:405-409

13. Louis DN, Perry A, Reifenberger G, von Deimling A, Figarella-Branger D, Cavenee WK et al (2016) The 2016 world health organization classification of tumors of the central nervous system: a summary. Acta Neuropathol 131:803-820

14. Tsien C, Galbán CJ, Chenevert TL, Johnson TD, Hamstra DA, Sundgren PC et al (2010) Parametric response map as an imaging biomarker to distinguish progression from pseudoprogression in high-grade glioma. J Clin Oncol 28:2293-2299

15. Gasparetto EL, Pawlak MA, Patel SH, Huse J, Woo JH, Krejza J et al (2009) Posttreatment recurrence of malignant brain neoplasm: accuracy of relative cerebral blood volume fraction in discriminating low from high malignant histologic volume fraction. Radiology 250:887-896

16. Rock JP, Scarpace L, Hearshen D, Gutierrez J, Fisher JL, Rosenblum M et al (2004) Associations among magnetic resonance spectroscopy, apparent diffusion coefficients, and image-guided histopathology with special attention to radiation necrosis. Neurosurgery 54:1111-1117 discussion 1117-1119

17. Garlan F, Laurent-Puig P, Sefrioui D, Siauve N, Didelot A, Sarafan-Vasseur N et al (2017) Early evaluation of circulating tumor DNA as marker of therapeutic efficacy in metastatic colorectal cancer patients (PLACOL study). Clin Cancer Res 23:5416-5425

18. Fribbens C, Garcia Murillas I, Beaney M, Hrebien S, O'Leary B, Kilburn L et al (2018) Tracking evolution of aromatase inhibitor resistance with circulating tumour DNA analysis in metastatic breast cancer. Ann Oncol 29:145-153

19. Chiu A, Ayub M, Dive C, Brady G, Miller CJ (2017) twoddpcr: an R/ bioconductor package and shiny app for droplet digital PCR analysis. Bioinformatics 33:2743-2745

20. Nabavizadeh SA, Ware JB, Guiry S, Nasrallah MP, Mays JJ, Till JE et al (2020) Imaging and histopathologic correlates of plasma cell-free DNA concentration and circulating tumor DNA in adult patients with newly diagnosed glioblastoma. Neurooncol Adv 2:vdaa016

21. Labussière M, Boisselier B, Mokhtari K, Di Stefano A-L, Rahimian A Rossetto M et al (2014) Combined analysis of TERT, EGFR, and IDH status defines distinct prognostic glioblastoma classes. Neurology 83:1200-1206

22. Mouliere F, Chandrananda D, Piskorz AM, Moore EK, Morris J, Ahlborn LB, et al. (2018) Enhanced detection of circulating tumor DNA by fragment size analysis. Sci Transl Med 10

23. Lun M, Lok E, Gautam S, Wu E, Wong ET (2011) The natural history of extracranial metastasis from glioblastoma multiforme. J Neurooncol 105:261-273

24. Beauchesne P (2011) Extra-neural metastases of malignant gliomas: myth or reality? Cancers (Basel) 3:461-477

25. Pietschmann S, von Bueren AO, Kerber MJ, Baumert BG, Kortmann RD, Müller K (2015) An individual patient data meta-analysis on characteristics, treatments and outcomes of glioblastoma/gliosarcoma patients with metastases outside of the central nervous system. PLoS ONE 10:e0121592

26. Pan C, Diplas BH, Chen X, Wu Y, Xiao X, Jiang L et al (2019) Molecular profiling of tumors of the brainstem by sequencing of CSF-derived circulating tumor DNA. Acta Neuropathol 137:297-306

27. De Mattos-Arruda L, Mayor R, Ng CKY, Weigelt B, Martínez-Ricarte F, Torrejon D et al (2015) Cerebrospinal fluid-derived circulating tumour DNA better represents the genomic alterations of brain tumours than plasma. Nat Commun 6:8839

28. Tie J, Kinde I, Wang Y, Wong HL, Roebert J, Christie M et al (2015) Circulating tumor DNA as an early marker of therapeutic response in patients with metastatic colorectal cancer. Ann Oncol 26:1715-1722

\section{Publisher's Note}

Springer Nature remains neutral with regard to jurisdictional claims in published maps and institutional affiliations.

Ready to submit your research? Choose BMC and benefit from

- fast, convenient online submission

- thorough peer review by experienced researchers in your field

- rapid publication on acceptance

- support for research data, including large and complex data types

- gold Open Access which fosters wider collaboration and increased citations

- maximum visibility for your research: over 100M website views per year

At BMC, research is always in progress.

Learn more biomedcentral.com/submissions 